\title{
A simple confidence interval for the difference between two normal population means with one variance unknown
}

\author{
Suparat Niwitpong \\ Department of Applied Statistics, Faculty of Applied Science, King Mongkut's University of \\ Technology North Bangkok, Bangkok 10800, Thailand \\ E-mail: suparatn@kmutnb.ac.th
}

\begin{abstract}
In this paper, asymptotic coverage probabilities and expected lengths of confidence intervals for the difference between the means of two normal populations with one variance unknown are derived. Monte Carlo simulations results indicate that our proposed confidence interval, which is easy to use, performs as well as the existing confidence intervals.
\end{abstract}

Keywords: confidence interval, coverage probability, expected length, normal means.

\section{Introduction}

Recently, confidence intervals for the difference between two normal population means have been investigated. it is known that a confidence interval based on the $t$-distribution with pooled sample variances is preferable when it is assumed that two population variances are equal; otherwise, the Welch-Satterthwaite (WS, hereafter) confidence interval is preferable, see e.g. Satterthwaite [1-2], Welch [3], Miao and Chiou [4], Moser et al. [5], Schechtman and Sherman [6] and references therein. Miao and Chiou [4] mentioned that the WS, Satterthwaite [1, 2], confidence interval also performs well, based on its coverage probability, in the case of two equal population variances. Niwitpong and Niwitpong [7] also derived coverage probabilities and expected lengths of confidence intervals for the difference between two normal means with a known ratio of variances. They revealed that the confidence interval based on $t$ statistic of Schechtman and Sherman [6] has a shorter expected length than that of the WS confidence interval when the ratio of variances is large.

A confidence interval for the difference of two normal population means with one variance unknown is also of interest. Maity and Sherman [8] described that this situation arises, for instance, when one is interested in comparing a standard treatment with a new treatment. A known variance comes from the standard treatment while an unknown variance comes from the new treatment.

Maity and Sherman found that their proposed $t$-test has more power than an existing Satterthwaite's test. Peng and Tong [9] proved that the t-test statistic with the number of degrees of freedom $v_{2}$ (see, section 2.2) of Maity and Sherman [8] is the biased estimator of the number of degrees of freedom. Hence, they proposed an unbiased degrees of freedom of the t-test statistic which is described in section 2.2. Peng and Tong [9] showed that the t-test statistic with the unbiased estimator of the number of degrees of freedom $v_{3}$ performs better that that of the Maity and Sherman's method especially when the variance of the unknown variance is large. In practice, both test statistics, however, need time to compute the number of degrees of freedom.

In this paper, we therefore, propose a simple and easy method to construct the confidence interval with one variance unknown as in Niwitpong [10]. We also proved the coverage probability and the expected length of each confidence interval in comparison with the WS confidence interval. The paper is organized as follows. Section 2 presents confidence intervals for the difference between two normal population means with one variance unknown. Coverage probabilities and expected lengths of confidence intervals in section 2 are derived in section 3 . Section 4 contains a discussion of the results and conclusions. 


\section{Confidence intervals for the difference of two normal population means}

Let $X_{1}, X_{2}, \ldots, X_{n}$ and $Y_{1}, Y_{2}, \ldots, Y_{m}$ be random samples from two independent normal distributions with means with means $\mu_{x}, \mu_{y}$ and standard deviations $\sigma_{x}$ and $\sigma_{y}$, respectively The sample means and variances for $X$ and $Y$ are also denoted as $\bar{X}, \bar{Y}, S_{X}^{2}$ and $S_{Y}^{2}$, respectively, where

$$
\bar{X}=\sum_{i=1}^{n} X_{i} / n, \bar{Y}=\sum_{j=1}^{m} Y_{j} / m, S_{x}^{2}=(n-1)^{-1} \sum_{i=1}^{n}\left(X_{i}-\bar{X}\right)^{2}, S_{y}^{2}=(m-1)^{-1} \sum_{j=1}^{m}\left(Y_{j}-\bar{Y}\right)^{2} .
$$

We are interested in 100(1- $\alpha) \%$ confidence interval for $\theta=\mu_{x}-\mu_{y}$ when one of the variance is unknown.

\subsection{The Confidence interval for $\theta$ based on Welch-Satterthwaite's method}

It is known that when the two variances differ, i.e. $\sigma_{x}^{2} \neq \sigma_{y}^{2}$, the confidence interval for $\theta$ is constructed using the test statistic $T_{1}$, as the pivot quantity,

$$
T_{1}=\frac{(\bar{X}-\bar{Y})-\left(\mu_{X}-\mu_{Y}\right)}{\sqrt{\frac{S_{X}^{2}}{n}+\frac{S_{Y}^{2}}{m}}} .
$$

It is also well-known that $T_{1}$ is approximately distributed as a $t$-distribution with degrees of freedom equal to

$$
v_{1}=\frac{(A+B)}{\frac{A^{2}}{n-1}+\frac{B^{2}}{m-1}}, A=\frac{S_{X}^{2}}{n}, B=\frac{S_{Y}^{2}}{m} .
$$

The $100(1-\alpha) \%$ confidence interval for $\theta$ is therefore

$$
C I_{W S}=\left[(\bar{X}-\bar{Y})-t_{1-\alpha / 2, v_{1}} \sqrt{\frac{S_{X}^{2}}{n}+\frac{S_{Y}^{2}}{m}},(\bar{X}-\bar{Y})+t_{1-\alpha / 2, v_{1}} \sqrt{\frac{S_{X}^{2}}{n}+\frac{S_{Y}^{2}}{m}}\right]
$$

where $t_{1-\alpha / 2, v_{1}}$ is the $(1-\alpha / 2)$ th percentile of $T_{1}$ distribution with $v_{1}$ degrees of freedom.

The confidence interval $C I_{W S}$ is known as the WS confidence interval.

\subsection{Existing confidence intervals for $\theta$ with one variance unknown}

Following Maity and Sherman [8], suppose $\sigma_{Y}^{2}$ is unknown, Maity and Sherman proposed the test statistic $T_{2}$,

$$
T_{2}=\frac{(\bar{X}-\bar{Y})-\left(\mu_{x}-\mu_{y}\right)}{\sqrt{\frac{\sigma_{x}^{2}}{n}+\frac{S_{y}^{2}}{m}}}
$$

of the hypothesis $H_{0}: \mu_{X}-\mu_{Y}=\Delta_{0}$ against the alternative hypothesis $H_{1}: \mu_{X}-\mu_{Y} \neq(<,>) \Delta_{0}$. By means of Monte Carlo simulation, they found that the test statistic $T_{2}$ has better power than the test statistic $T_{1}$ when one variance is unknown. However, they did not study the confidence interval for $\theta$ using the pivotal test statistic $T_{2}$.

As a result, it is of interest to construct the confidence interval for $\theta$ when one variance is unknown. Niwitpong [10] proposed the confidence interval for $\theta$ using the pivotal quantity $T_{2}$ with $v_{2}$ degrees of freedom where

$$
v_{2}=\frac{\left(\frac{\sigma_{X}^{2}}{n}+\frac{S_{Y}^{2}}{m}\right)^{2}}{\frac{\left(S_{Y}^{2} / m\right)^{2}}{m-1}} .
$$

Maity and Sherman [8] pointed out that the test statistic $T_{2}$ has an approximate $t$-distribution with $v_{2}$ degrees of freedom. Define $t_{1-\alpha / 2, v_{2}}$ be the $(1-\alpha / 2) t h$ percentile of the $t$-distribution with

$v_{2}$ Degrees of freedom, such that 


$$
\operatorname{Pr}\left[(\bar{X}-\bar{Y})-t_{1-\alpha / 2, v_{2}} \sqrt{\frac{\sigma_{x}^{2}}{n}+\frac{S_{y}^{2}}{m}}<\theta<(\bar{X}-\bar{Y})+t_{1-\alpha / 2, v_{2}} \sqrt{\frac{\sigma_{x}^{2}}{n}+\frac{S_{y}^{2}}{m}}\right]=1-\alpha .
$$

Therefore, $100(1-\alpha) \%$ confidence interval for $\theta$ is

$$
C I_{M S}=\left[(\bar{X}-\bar{Y})-t_{1-\alpha / 2, v_{2}} \sqrt{\frac{\sigma_{X}^{2}}{n}+\frac{S_{Y}^{2}}{m}},(\bar{X}-\bar{Y})+t_{1-\alpha / 2, v_{2}} \sqrt{\frac{\sigma_{X}^{2}}{n}+\frac{S_{Y}^{2}}{m}}\right] .
$$

Niwitpong [10] derived the coverage probability and the expected length of the confidence intervals $C I_{W S}$ and $C I_{M S}$ for $\theta$, when one variance unknown. She found that coverage probabilities of both confidence intervals $C I_{W S}$ and $C I_{M S}$ are almost the same but $C I_{M S}$ has a smaller expected length when the unknown variance is small.

Peng and Tong [9] showed that the number of degrees of freedom $v_{2}$ of the test statistic $T_{2}$ is a positive biased estimator of $v$ where

$$
v=\frac{\left(\frac{\sigma_{X}^{2}}{n}+\frac{\sigma_{Y}^{2}}{m}\right)^{2}}{\frac{\left(\sigma_{Y}^{2} / m\right)^{2}}{m-1}} .
$$

Hence, they proposed an unbiased estimator of $v$ which is

$$
v_{3}=(m-1)\left\{\frac{m^{3}(m-3)(m-5) \sigma_{x}^{2}}{n^{2}(n-1)^{2}} \frac{1}{S_{y}^{2}}+\frac{2 m(m-3) \sigma_{x}^{2}}{n(n-1)} \frac{1}{S_{y}^{2}}+1\right\}
$$

for the test statistic $T_{2}$.

By means of simulation, Peng and Tong [9] showed that the test statistic $T_{2}$ with degrees of freedom $v_{3}$ can control type I error better than that of the test statistic $T_{2}$ with degrees of freedom $v_{2}$, especially when $\sigma_{Y}^{2}$ is large.

Similarly to $C I_{M S}$, we construct a new confidence interval for $\theta=\mu_{x}-\mu_{y}$ by using the pivotal statistic $T_{2}$ with degrees of freedom $v_{3}$, and this confidence interval is denoted as $C I_{J T}$, where

$$
C I_{J T}=\left[(\bar{X}-\bar{Y})-t_{1-\alpha / 2, v_{3}} \sqrt{\frac{\sigma_{X}^{2}}{n}+\frac{S_{Y}^{2}}{m}},(\bar{X}-\bar{Y})+t_{1-\alpha / 2, v_{3}} \sqrt{\frac{\sigma_{X}^{2}}{n}+\frac{S_{Y}^{2}}{m}}\right] .
$$

\subsection{Proposed confidence interval for $\theta$ with one variance unknown}

It is argued that, in practice, confidence intervals $C I_{M S}$ and $C I_{J T}$ are not easy to use. This is due to the calculation of the number of degrees of freedom $v_{2}$ and $v_{3}$. We now propose an easy method, based on Zou et al. [11-12], to construct a new confidence interval for $\theta$ with one variance unknown. Our proposed confidence interval constructed using the test statistics $\mathrm{Z}$ (standard normal distribution) and $\mathrm{T}$ with degrees of freedom $\mathrm{m}-1$.

Zou et al. [11-12] proposed the method called "The Method of Variance Estimates Recovery (MOVER)" to construct confidence intervals for lognormal and normal data. Their strategy is to recover variance estimates from confidence interval of each parameter and then approximate confidence intervals for functions of parameters by using the central limit theorem. A general approach to construct two-sided confidence interval for $\theta_{1}+\left(-\theta_{2}\right)$ is $[L, U]$ where

$$
L=\left(\hat{\theta}_{1}-\hat{\theta}_{2}\right)-\sqrt{\left(\hat{\theta}_{1}-l_{1}\right)^{2}+\left(u_{2}-\hat{\theta}_{2}\right)^{2}}
$$

and

$$
U=\left(\hat{\theta}_{1}-\hat{\theta}_{2}\right)-\sqrt{\left(u_{1}-\hat{\theta}_{1}\right)^{2}+\left(\hat{\theta}_{2}-l_{2}\right)^{2}},
$$

$\hat{\theta}_{1}, \hat{\theta}_{2}$ are respectively estimators of $\theta_{i}, i=1,2$ and $\theta_{i} \in\left(l_{i}, u_{i}\right)$.

For $\left(l_{i}, u_{i}\right),(i=1,2)$, setting $\theta_{1}=\mu_{x}$ and $\theta_{2}=\mu_{y}$, it is known that confidence intervals for each $\theta_{i}, i=1,2$ are respectively

$$
\left(l_{1}, u_{1}\right)=\left(\bar{X}-Z_{1-\alpha / 2} \frac{\sigma_{x}}{\sqrt{n}}, \bar{X}+Z_{1-\alpha / 2} \frac{\sigma_{x}}{\sqrt{n}}\right)
$$




$$
\left(l_{2}, u_{2}\right)=\left(\bar{Y}-t_{1-\alpha / 2, m-1} \sqrt{\frac{S_{y}^{2}}{m}}, \bar{Y}+t_{1-\alpha / 2, m-1} \sqrt{\frac{S_{y}^{2}}{m}}\right) .
$$

Putting $\hat{\theta}_{1}=\bar{X}$, and $\hat{\theta}_{2}=\bar{Y}, l_{1}=\bar{X}-Z_{1-\alpha / 2} \frac{\sigma_{x}}{\sqrt{n}}, u_{1}=\bar{X}+Z_{1-\alpha / 2} \frac{\sigma_{x}}{\sqrt{n}} \quad$ and

$$
\begin{gathered}
l_{2}=\bar{Y}-t_{1-\alpha / 2} \frac{S_{y}}{\sqrt{m}}, u_{2}=\bar{Y}+t_{1-\alpha / 2} \frac{S_{y}}{\sqrt{m}} \text { in }[L, U] \text {, we have } \\
L^{\prime}=\bar{X}-\bar{Y}-\sqrt{\left(\bar{X}-\bar{X}+Z_{1-\alpha / 2} \frac{\sigma_{x}}{\sqrt{n}}\right)^{2}+\left(\bar{Y}+t_{1-\alpha / 2, m-1} \frac{S_{y}}{\sqrt{m}}-\bar{Y}\right)^{2}} \\
=\bar{X}-\bar{Y}-\sqrt{Z_{1-\alpha / 2}^{2} \frac{\sigma_{x}^{2}}{n}+t_{1-\alpha / 2, m-1}^{2} \frac{S_{y}^{2}}{m}} \\
\begin{aligned}
U^{\prime}=\bar{X}-\bar{Y}+\sqrt{\left(\bar{X}+Z_{1-\alpha / 2} \frac{\sigma_{x}}{\sqrt{n}}-\bar{X}\right)^{2}+\left(\bar{Y}-\bar{Y}+t_{1-\alpha / 2, m-1} \frac{S_{y}}{\sqrt{m}}\right)^{2}} \\
=\bar{X}-\bar{Y}+\sqrt{Z_{1-\alpha / 2}^{2} \frac{\sigma_{x}^{2}}{n}+t_{1-\alpha / 2, m-1}^{2} \frac{S_{y}^{2}}{m} .}
\end{aligned}
\end{gathered}
$$

A new confidence interval for $\theta=\mu_{x}-\mu_{y}$ with unknown $\sigma_{Y}^{2}$ is therefore a confidence interval $C I_{N}=\left[L^{\prime}, U^{\prime}\right]$ where

$$
C I_{N}=\left[\bar{X}-\bar{Y}-\sqrt{Z_{1-\alpha / 2}^{2} \frac{\sigma_{x}^{2}}{n}+t_{1-\alpha / 2, m-1}^{2} \frac{S_{y}^{2}}{m}}, \bar{X}-\bar{Y}+\sqrt{Z_{1-\alpha / 2}^{2} \frac{\sigma_{x}^{2}}{n}+t_{1-\alpha / 2, m-1}^{2} \frac{S_{y}^{2}}{m}}\right] .
$$

We evaluate these confidence intervals i.e. $C I_{W S}, C I_{M S}, C I_{J T}$ and $C I_{N}$ using their coverage probabilities and expected lengths which are derived in the next section. Generally, we prefer a confidence interval with minimum coverage probability equal to a pre-specified value 1- $\alpha$ and with a shorter expected length.

\section{Main results: Coverage probabilities and expected lengths of confidence intervals for $\theta$ with one variance unknown}

In this section, the coverage probabilities and the expected lengths of confidence intervals $C I_{W S}, C I_{M S}, C I_{J T}$ and $C I_{N}$ are derived.

Theorem 1 :( Niwitpong and Niwitpong [7]) The coverage probability and the expected length of $C I_{W S}$ when one variance $\left(\sigma_{Y}^{2}\right)$ is unknown are respectively

$$
\begin{aligned}
& E\left[\Phi\left(w_{1}\right)-\Phi\left(-w_{1}\right)\right] \text { and } \\
& \left\{\begin{array}{l}
2 d \zeta \sqrt{s_{1}} F\left[\frac{-1}{2}, \frac{m-1}{2}, \frac{m+n-2}{2}, \frac{s_{1}-s_{2}}{s_{1}}\right] ; \text { if } s_{2}<2 s_{1}, \\
2 d \zeta \sqrt{s_{2}} F\left[\frac{-1}{2}, \frac{n-1}{2}, \frac{m+n-2}{2}, \frac{s_{2}-s_{1}}{s_{2}}\right] ; \text { if } 2 s_{1} \leq s_{2}
\end{array}\right. \\
& \text { where } \quad w_{1}=t_{1-\frac{\alpha}{2}, v} \frac{\left(\frac{S_{X}^{2}}{n}+\frac{S_{Y}^{2}}{m}\right)^{1 / 2}}{\left(\frac{\sigma_{X}^{2}}{n}+\frac{\sigma_{Y}^{2}}{m}\right)^{1 / 2},}
\end{aligned}
$$


$\Phi[$.$] is the cumulative distribution function of N(0,1)$,

$$
s_{1}=\frac{\sigma_{X}^{2}}{n(n-1)}, s_{2}=\frac{\sigma_{Y}^{2}}{m(m-1)}, \varsigma=\frac{\sqrt{2} \Gamma\left(\frac{m+n-1}{2}\right)}{\Gamma\left(\frac{m+n-2}{2}\right)},
$$

$F(a, b, c ; k)$ is the hypergeometric function defined by $F(a, b, c ; k)=1+\frac{a b}{c} \frac{k}{1 !}+\frac{a(a+1) b(b+1)}{c(c+1)} \frac{k^{2}}{2 !}+\ldots$ and $\Gamma[$.$] is the gamma function.$

Proof: See Niwitpong and Niwitpong [7].

Theorem 2: ( Niwitpong [10]) The coverage probability and the expected length of $C I_{M S}$ with one variance unknown $\left(\sigma_{Y}^{2}\right)$ are respectively

$$
\begin{aligned}
& E\left[\Phi\left(w_{2}\right)-\Phi\left(-w_{2}\right)\right] \text { and } 2 t_{1-\alpha / 2, v_{2}}\left(\frac{\sigma_{X}^{2}}{n}+\frac{\sigma_{Y}^{2}}{m}\right)^{1 / 2} \\
& \text { where } \quad w_{2}=t_{1-\alpha / 2, v_{2}} \frac{\sqrt{\frac{\sigma_{X}^{2}}{n}+\frac{S_{Y}^{2}}{m}}}{\sqrt{\frac{\sigma_{X}^{2}}{n}+\frac{\sigma_{Y}^{2}}{m}}} .
\end{aligned}
$$

Proof : See Niwitpong [10]

Theorem 3: The coverage probability and the expected length of $C I_{J T}$ with one variance unknown ( $\left.\sigma_{Y}^{2}\right)$ are respectively

$$
E\left[\Phi\left(w_{3}\right)-\Phi\left(-w_{3}\right)\right] \text { and } 2 t_{1-\alpha / 2, v_{3}}\left(\frac{\sigma_{X}^{2}}{n}+\frac{\sigma_{Y}^{2}}{m}\right)^{1 / 2}
$$

where

$$
w_{3}=t_{1-\alpha / 2, v_{3}} \frac{\sqrt{\frac{\sigma_{X}^{2}}{n}+\frac{S_{Y}^{2}}{m}}}{\sqrt{\frac{\sigma_{X}^{2}}{n}+\frac{\sigma_{Y}^{2}}{m}}} .
$$

Proof: It is straightforward to see that the coverage probability of $C I_{J T}$ is

$$
\begin{aligned}
& 1-\alpha=\operatorname{Pr}\left((\bar{X}-\bar{Y})-d_{1} \sqrt{\frac{\sigma_{X}^{2}}{n}+\frac{S_{Y}^{2}}{m}} \leq \theta \leq(\bar{X}-\bar{Y})+d_{1} \sqrt{\frac{\sigma_{X}^{2}}{n}+\frac{S_{Y}^{2}}{m}}\right), \quad d_{1}=t_{1-\alpha / 2, v_{3}} \\
& =\operatorname{Pr}\left(-d_{1} \sqrt{\frac{\sigma_{X}^{2}}{n}+\frac{S_{Y}^{2}}{m}} \leq \theta-(\bar{X}-\bar{Y}) \leq d_{1} \sqrt{\frac{\sigma_{X}^{2}}{n}+\frac{S_{Y}^{2}}{m}}\right) \\
& =\operatorname{Pr}\left(-d_{1} \frac{\sqrt{\frac{\sigma_{X}^{2}}{n}+\frac{S_{Y}^{2}}{m}}}{\sqrt{\frac{\sigma_{X}^{2}}{n}+\frac{\sigma_{Y}^{2}}{m}}} \leq Z \leq d_{1} \frac{\sqrt{\frac{\sigma_{X}^{2}}{n}+\frac{S_{Y}^{2}}{m}}}{\sqrt{\frac{\sigma_{X}^{2}}{n}+\frac{\sigma_{Y}^{2}}{m}}}\right) \\
& =E\left[I_{\left\{-w_{3}<Z<w_{3}\right\}}(\tau)\right], I_{\left\{-w_{3}<Z<w_{3}\right\}}(\tau)=\left\{\begin{array}{l}
1, \tau \in\left\{-w_{3}<Z<w_{3}\right\} \\
0, \text { otherwise }
\end{array}\right.
\end{aligned}
$$




$$
\begin{aligned}
& =E\left[E\left[I_{\left\{-w_{3}<Z<w_{3}\right\}}(\tau)\right] \mid S_{Y}^{2}\right] \\
& =E\left[\Phi\left(w_{3}\right)-\Phi\left(-w_{3}\right)\right] .
\end{aligned}
$$

The length of $C I_{J T}$ is $2 d_{1} \sqrt{\frac{\sigma_{X}^{2}}{n}+\frac{S_{Y}^{2}}{m}}$ and the expected length of $C I_{J T}$ is

$$
E\left(2 d_{1} \sqrt{\frac{\sigma_{X}^{2}}{n}+\frac{S_{Y}^{2}}{m}}\right)=2 d_{1} E\left(\sqrt{\frac{\sigma_{X}^{2}}{n}+\frac{S_{Y}^{2}}{m}}\right) \leq 2 d_{1}\left(E\left(\frac{\sigma_{X}^{2}}{n}+\frac{S_{Y}^{2}}{m}\right)\right)^{1 / 2},
$$

using Jensen's inequality and (Niwitpong [10], Lemma 1), we have $E_{r}(f(r)) \leq f(E(r))$

$$
\begin{aligned}
& =2 d_{1}\left(\frac{\sigma_{X}^{2}}{n}+\frac{\sigma_{Y}^{2}}{m(m-1)} E\left(\frac{(m-1) S_{Y}^{2}}{\sigma_{Y}^{2}}\right)\right)^{1 / 2} \\
& =2 d_{1}\left(\frac{\sigma_{X}^{2}}{n}+\frac{\sigma_{Y}^{2}}{m(m-1)} E\left(U_{2}\right)\right)^{1 / 2}, \text { since } U_{2} \sim \chi_{m-1}^{2} \\
& =2 d_{1}\left(\frac{\sigma_{X}^{2}}{n}+\frac{\sigma_{Y}^{2}}{m(m-1)}(m-1)\right)^{1 / 2}, E\left(U_{2}\right)=m-1 \\
& =2 d_{1}\left(\frac{\sigma_{X}^{2}}{n}+\frac{\sigma_{Y}^{2}}{m}\right)^{1 / 2} .
\end{aligned}
$$

This ends the proof.

Theorem 4: The coverage probability and the expected length of $C I_{N}$ with one variance unknown $\left(\sigma_{Y}^{2}\right)$ are respectively

$$
E\left[\Phi\left(w_{5}\right)-\Phi\left(-w_{5}\right)\right] \quad \text { and } \quad 2\left(\frac{Z_{1-\alpha / 2}^{2} \sigma_{x}^{2}}{n}+\frac{t_{1-\alpha / 2, v_{3}}^{2} \sigma_{y}^{2}}{m}\right)^{\frac{1}{2}}
$$

where

$$
w_{5}=\frac{\sqrt{\frac{Z_{1-\alpha / 2}^{2} \sigma_{x}^{2}}{n}+\frac{t_{1-\alpha / 2, v_{3}}^{2} \sigma_{y}^{2}}{m}}}{\sqrt{\frac{\sigma_{X}^{2}}{n}+\frac{\sigma_{Y}^{2}}{m}}} .
$$

Proof: Let $C_{1}=Z_{1-\alpha / 2}^{2}, C_{2}=t_{1-\alpha / 2, v_{3}}^{2}$, the coverage probability of $C I_{N}$ is

$$
\begin{aligned}
1-\alpha=\operatorname{Pr}\left(\theta \in C I_{N}\right) & =P\left(\theta \in\left(L^{\prime}, U^{\prime}\right)\right) \\
& =P\left(\bar{X}-\bar{Y}-\sqrt{C_{1} \frac{\sigma_{x}^{2}}{n}+C_{2} \frac{S_{y}^{2}}{m}}<\theta<\bar{X}-\bar{Y}+\sqrt{C_{1} \frac{\sigma_{x}^{2}}{n}+C_{2} \frac{S_{y}^{2}}{m}}\right) \\
& =P\left(-\sqrt{C_{1} \frac{\sigma_{x}^{2}}{n}+C_{2} \frac{S_{y}^{2}}{m}}<\theta-(\bar{X}-\bar{Y})<+\sqrt{C_{1} \frac{\sigma_{x}^{2}}{n}+C_{2} \frac{S_{y}^{2}}{m}}\right) \\
& =P\left(-w_{4}<\theta-(\bar{X}-\bar{Y})<w_{4}\right), w_{4}=\sqrt{C_{1} \frac{\sigma_{x}^{2}}{n}+C_{2} \frac{S_{y}^{2}}{m}} \\
& =P\left(w_{4}>(\bar{X}-\bar{Y})-\theta>-w_{4}\right) \\
& =P\left(-\frac{w_{4}}{\sqrt{A}}<\frac{(\bar{X}-\bar{Y})-\theta}{\sqrt{A}}<\frac{w_{4}}{\sqrt{A}}\right), A=\frac{\sigma_{x}^{2}}{n}+\frac{\sigma_{y}^{2}}{m}
\end{aligned}
$$




$$
\begin{aligned}
& =P\left(-w_{5}<Z<w_{5}\right), w_{5}=\frac{w_{4}}{\sqrt{A}} \\
& =E\left[I_{\left\{-w_{5}<Z<w_{5}\right\}}(\xi)\right], I_{\left\{-w_{5}<Z<w_{5}\right\}}(\xi)=\left\{\begin{array}{l}
1, \xi \in\left\{-w_{5}<Z<w_{5}\right\} \\
0, \text { otherwise }
\end{array}\right. \\
& =E\left[E\left[I_{\left\{-w_{5}<Z<w_{5}\right\}}(\xi)\right] \mid S_{Y}^{2}\right]=E\left[\Phi\left(w_{5}\right)-\Phi\left(-w_{5}\right)\right]
\end{aligned}
$$

where $Z \sim N(0,1)$.

The Length of $C I_{C}$ is

$$
\begin{aligned}
E\left(2 \sqrt{C_{1} \frac{\sigma_{x}^{2}}{n}+C_{2} \frac{S_{y}^{2}}{m}}\right) & =2 E\left(C_{1} \frac{\sigma_{x}^{2}}{n}+C_{2} \frac{S_{y}^{2}}{m}\right)^{\frac{1}{2}} \\
& \leq 2\left(E\left(C_{1} \frac{\sigma_{x}^{2}}{n}+C_{2} \frac{S_{y}^{2}}{m}\right)\right)^{\frac{1}{2}} \\
& =2\left(C_{1} \frac{\sigma_{x}^{2}}{n}+C_{2} E\left(\frac{(m-1)}{(m-1)}\right) \frac{S_{y}^{2}}{m} \frac{\sigma_{y}^{2}}{\sigma_{y}^{2}}\right)^{\frac{1}{2}} \\
& =2\left(C_{1} \frac{\sigma_{x}^{2}}{n}+C_{2} \frac{\sigma_{y}^{2}}{m(m-1)} E\left(\chi_{m-1}^{2}\right)\right)^{\frac{1}{2}} \\
& =2\left(C_{1} \frac{\sigma_{x}^{2}}{n}+\frac{C_{2} \sigma_{y}^{2}}{m(m-1)}(m-1)\right)^{\frac{1}{2}} \\
& =2\left(\frac{C_{1} \sigma_{x}^{2}}{n}+\frac{C_{2} \sigma_{y}^{2}}{m}\right)^{\frac{1}{2}} .
\end{aligned}
$$

This ends the proof.

\section{Discussion and conclusions}

We proposed, in this paper, the new confidence interval for the difference between two normal population means with one variance unknown, $C I_{N}$. We derived coverage probability of the new confidence interval compared to existing confidence intervals: $C I_{W S}, C I_{M S}$ and $C I_{J T}$. Theorems 1-4 show that all confidence intervals have coverage probability $1-\alpha$, for large samples and $\alpha$ is a level of significance for all test statistics in this paper. The expected length of the confidence interval $C I_{J T}$ is as short as the confidence interval $C I_{M S}$ and the expected length of our proposed confidence interval $C I_{N}$ is at least shorter than that of the confidence interval $C I_{J T}$ but our proposed confidence interval is simple to use more than those of existing confidence intervals which are needed to compute the number of degrees of freedoms $v_{2}$ and $v_{3}$.

\section{Acknowledgements}

The author thanks the financial support from Faculty of Applied Sciences, King Mongkut's University of Technology North Bangkok. 


\section{References}

[1] F.E. Satterthwaite, "Synthesis of variance," Psychometrika, vol. 6, pp. 309-316. 1941

[2] F.E. Satterthwaite, "An appoximate distribution of estimates of variance Components," Biometric Bulletin, vol. 6, pp. 110-114, 1946.

[3] B.L. Welch, "The significance of the difference between two means when the Population variances are unequal," Biometric, vol. 34, pp. 2835,1938 .

[4] W. Miao and P. Chiou, "Confidence intervals for the difference between two means," Computational Statistics \& Data analysis, vol. 52, pp. 2238-2248, 2008.

[5] B.K Moser, G.R. Stevens, and C.L. Watts, "The two sample t-test versus Satterthwaite's approximation F- test," Communications in Statistics: Theory and Methods, vol.18, pp. 3963-3975, 1989.

[6] E. Schechtman and M. Sherman, "The two-sample t-test with a known ratio of Variances," Statistical Methodology, vol. 4, pp. 508-514, 2007.

[7] S. Niwitpong and S. Niwitpong, "Confidence interval for the difference of two normal population means with a known ratio of variances," Applied Mathematical Sciences, vol. 4, no. 8, pp.347-359, 2010.

[8] A. Maity, and M. Sherman, "The two sample t-test with one variance unknown," The American Statistician, vol. 60, pp.163-166, 2006.

[9] L. Peng and T. Tong, "A note on a two-sample T test with one variance unknown,"Statistical Methodology, vol. 8, pp. 528-534, 2011.

[10] S. Niwitpong, "Confidence intervals for the difference of two normal population means with one variance unknown," Thailand Statistician, vol. 7, no. 2, pp. 132-147, 2009.

[11] G.Y. Zou, C.Y. Huo, and J. Taleban, "Simple confidence intervals for lognormal means and their differences with environmental Applications," Environ metrics, vol. 20, pp. 172-180, 2009

[12] G.Y. Zou, J. Taleban, and C.Y. Huo, "Confidence interval estimation for lognormal data with application to health economics," Computational Statistics \& Data Analysis, vol. 53, pp. 3755-3764, 2009. 